\title{
How valid are old species lists? How archived samples can be used to update Ephemeroptera biodiversity information for northern Canada
}

\author{
Donna J. Giberson, ${ }^{1}$ Steven K. Burian
}

\begin{abstract}
Broad-scale aquatic insect ecological studies are an important potential source of biodiversity information, though taxa lists may contain outdated names or be incompletely or incorrectly identified. We re-examined over 12000 archived Ephemeroptera (mayfly) specimens from a large environmental assessment project (Mackenzie Valley pipeline study) in Yukon and the Northwest Territories, Canada (1971-1973) and compared the results to data from five recent (post-2000) collecting expeditions. Our goals were to update the species list for Ephemeroptera for Yukon and the Northwest Territories, and to evaluate the benefits of retaining and re-examining ecological samples to improve regional biodiversity information, particularly in isolated or inaccessible areas. The original pipeline study specimen labels reported 17 species in 25 genera for the combined Yukon and Northwest Territories samples, of which six species and 15 genera are still valid. Re-examination of specimens resulted in 45 species in 29 genera, with 14 and seven newly recorded species for Northwest Territories and Yukon, respectively. The recent collecting resulted in 50 species, 29 of which were different from the pipeline study, and five of which were new territorial records (Northwest Territories: four species; Yukon: one species). Re-examination of archived ecological specimens provides a cost-effective way to update regional biodiversity information.
\end{abstract}

\section{Introduction}

Regional species lists may be used to prepare species status assessment reports and to inform conservation efforts and environmental assessment studies (Catling et al. 2009; Robinson et al. 2016), but time and effort is needed to keep them up to date. For insects, including aquatic insects, these lists may be compiled from a combination of new sampling and checklists from published sources (e.g., Robinson et al. 2016). The Biological Survey of Canada (BSC) has been facilitating collection of species information in Canada for 40 years (since 1977; Danks 2016) and in that time, taxonomic tools for identification of organisms have advanced greatly. These include advances in online databases and geographic tools, improved access to new taxonomic revisions and keys as well as older taxonomic literature (see discussions in Guerra García et al. 2008; Tancoigne and Dubois 2013) and molecular methods like DNA barcoding (e.g., Ball et al. 2005; Webb et al. 2012). Although new sampling is usually preferred when carrying out regional biological surveys, the cost of such surveys may be prohibitive (Robinson et al. 2016), particularly for isolated areas such as the Canadian North (Cordero et al. 2016). Where available, ecological and species survey data from the literature can provide valuable baseline data and extend the information on species in a cost-effective manner (e.g., Robinson et al. 2016). How useful are these lists for Ephemeroptera species, and what factors must be considered to assess their use?

Challenges to using published species lists (e.g., from ecological surveys) generally focus on data quality (Stribling et al. 2003), including questions about correct identifications and

Received 6 March 2017. Accepted 19 April 2017. First published online 20 July 2017.

D.J. Giberson, ${ }^{1}$ Department of Biology, University of Prince Edward Island, 550 University Avenue, Charlottetown, Prince Edward Island, C1A 4P3, Canada

S.K. Burian, Department of Biology, Southern Connecticut State University, 501 Crescent Street, New Haven, Connecticut, 06515, United States of America

${ }^{1}$ Corresponding author (e-mail: giberson@upei.ca).

Subject editor: Héctor Cárcamo

doi:10.4039/tce.2017.27 
validity of older lists following taxonomic revisions (Patterson et al. 2016). Further, ecological studies may not identify specimens to species (due to a combination of resources and inappropriate life stages for identification), so the data are limited in their use for species assessments (Stribling et al. 2003). Some discrepancies in species lists can be resolved easily, simply by updating names that have been synonymised or transferred to different genera. However, taxa that have undergone significant revision, such as the Ephemeroptera family Baetidae, include new species and many new combinations as well as synonymies. For example, since the publication of the first comprehensive key to Baetis Leach species by Morihara and McCafferty (1979), several genera have been split from Baetis (e.g., McCafferty and Waltz 1995; McCafferty et al. 2008) and species from other baetid genera have been reassigned to several genera, including Baetis (McCafferty and Waltz 1990). The most recent key to Baetis species (Wiersema et al. 2004) is also out of date, as new species descriptions and synonymies continue to be published (e.g., Webb 2013; Jacobus and Wiersema 2014). Although historical studies provide a rich source of biodiversity information (and may also include important ecological context for species occurrences), specimens may need to be re-examined to ensure accurate and valid species lists from the study. Re-examination of material from historical studies also assumes that specimens have been archived in accessible collections, stored in a fashion that prevents or reduces deterioration, and are associated with readable label information.

The extensive Mackenzie River Pipeline study of 1971-1973 (Brunskill et al. 1973) provides a good example of an historical study, which can be used to update regional biodiversity information for a poorly known region in the Canadian north. The study was designed to examine potential impacts of construction and operation of an oil pipeline along the Mackenzie River, and resulted in thousands of aquatic insect samples from otherwise inaccessible and isolated areas being archived in governmentheld collections. Taxa lists from the study were published in Wiens et al. (1975) and Cobb and Flannagan (1980), but they list relatively few Ephemeroptera species and include many problematic generic names. In this study, we explore the benefits and challenges of updating the regional
Ephemeroptera species list for the Northwest Territories and northern Yukon by re-examining archived specimens. We also compare the benefits (in terms of species composition and diversity information) from the archived specimens to data from more recent sampling in the region.

\section{Methods}

Specimens examined for this study came from two sources: (1) historically archived collections from the Mackenzie Pipeline study (Porcupine River Drainage) in northern Yukon, and Mackenzie Valley tributaries in Northwest Territories (Brunskill et al. 1973; Wiens et al. 1975) and (2) from more recent collecting in Yukon in 2006, along the Horton and Thelon rivers (described in Currie et al. 2000, 2002, respectively), Mackenzie River tributaries (Rempel and Gill 2010; Vinke et al. 2015), and additional sites in Yukon and near Yellowknife, Norman Wells and Banks Island, Northwest Territories (Cordero et al. 2016) (Fig. 1).

Archived Mackenzie River pipeline study samples: The Mackenzie River study was carried out by researchers from the Freshwater Institute of Fisheries and Oceans Canada (Winnipeg, Manitoba, Canada), and methods and collecting localities are described in Brunskill et al. (1973). Aquatic invertebrates were collected from rivers, streams, and lakes along proposed pipeline routes from northern Yukon (Porcupine River Drainage) and the Mackenzie River Delta, to the southern portions of the Mackenzie River near Norman Wells and Fort Simpson, Northwest Territories (Fig. 1). Their goal was to assess taxonomic composition, seasonal and spatial diversity and abundance, effects of sediment addition on benthos, and effects of oil on benthos. The diversity of habitats and many study questions resulted in a wide diversity of sampling methods including Surber and kick sampling (small streams and river edges), drift nets and artificial substrate boxes (small-sized to medium-sized streams), floating baskets (large rivers), Ekman dredges (large rivers, lakes, ponds), dip nets (lake and river littoral samples), and pan traps (passive streamside adult collection at all sites). Samples were collected in all seasons, preserved in formalin, and later washed into $70 \%$ ethanol for sorting and identification. Samples were sent to the Biosystematics Research Institute in Ottawa, Ontario, Canada (now the 
Fig. 1. Yukon and Northwest Territories, showing locations of study areas sampled in this study. Open rectangles indicate the study areas from the 1971-1973 Mackenzie Valley pipeline study, and closed circles indicate the sampling sites visited in collecting trips since 2000. Tributaries and the wadeable sections of the main rivers were sampled along the entire river corridor in 2005 and 2006 for the Mackenzie River by L. Rempel (Rempel and Gill 2010) and for the Horton and Thelon rivers (Currie et al. 2000, 2002), so individual sampling sites are not indicated for these studies. Southern Yukon sites were sampled in 2006, and Ogilvie Mountain sites (along the Dempster Highway) were sampled in 2006 and again in 2010 by Cordero et al. (2016). Inset: map of Canada showing study region. AK, Alaska; YK, Yukon; NWT, Northwest Territories; NU, Nunuvut.

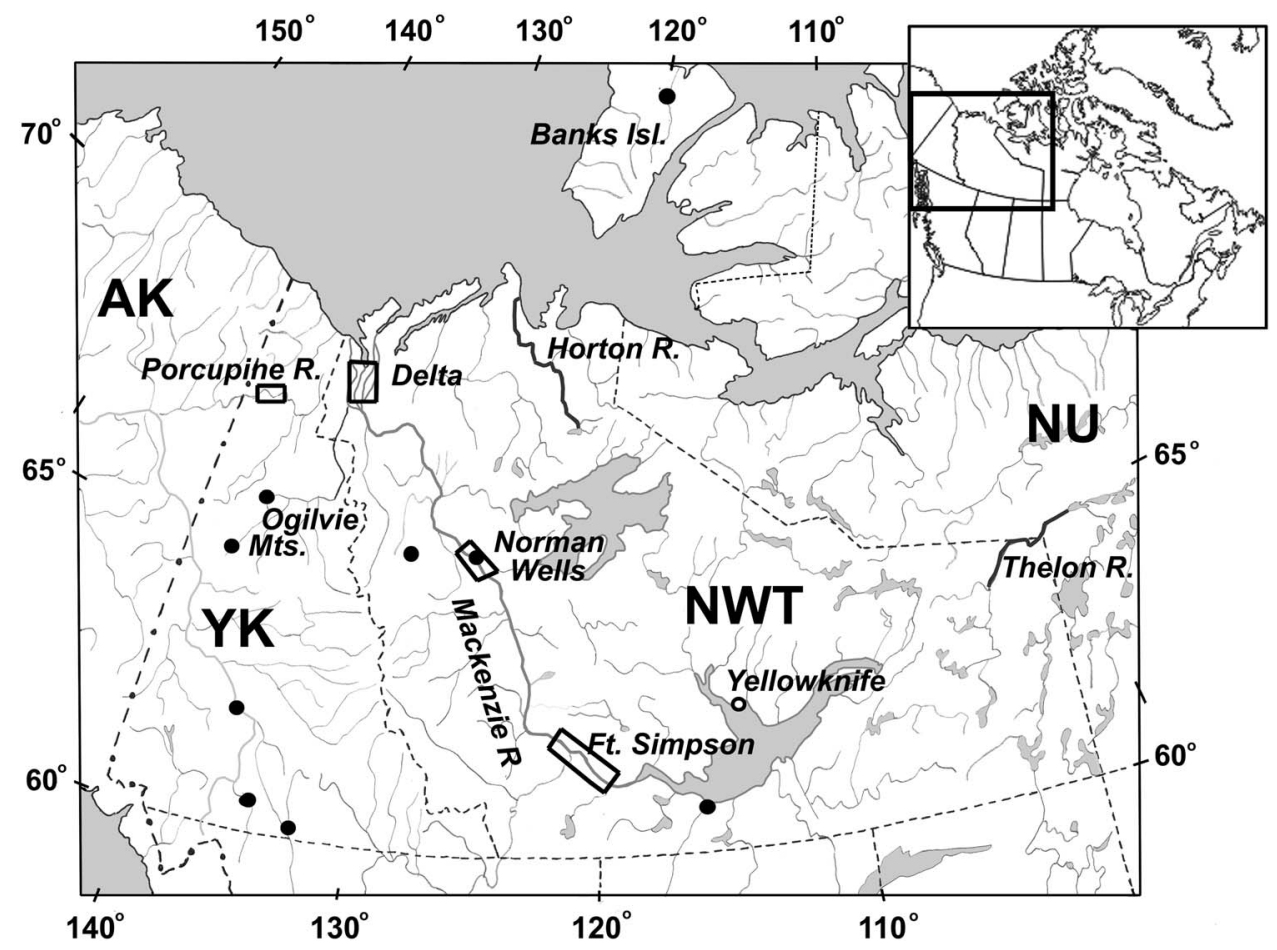

Canadian National Collection of Insects) for identification, and a subset of these were retained at the Canadian National Collection of Insects. Remaining vials were returned to the Freshwater Institute. They were then archived in storage until 2009, when pressure to clean out the vial room to make room for other uses prompted re-examination of the specimens for this study. In 2010, Mackenzie Study Ephemeroptera vials from Freshwater Institute and the Canadian National Collection of Insects were combined to give $\sim 2930$ vials representing 588 different sites and dates for re-examination. Some vials were broken or misplaced over the years, and others had dried, but 12215 specimens were available for re-examination for this study.

Recent collecting in Yukon and Northwest Territories: The more recent collections were garnered from five collecting trips/studies spanning the period between 2000 and 2011 (Fig. 1).

(1) Barrenland rivers in Northwest Territories, 2000-2002: The Horton and Thelon rivers (and their tributaries) were sampled in summer (June-July) 2000 and 2002, respectively, by travelling down them by canoe, and stopping at intervals to sample the main rivers and tributaries (Currie et al. 2000, 2002). Insects were collected in wadeable areas using kick/dip nets and along the stream edges using an aerial net, rock searches, and Malaise trapping. Mature specimens were sorted visually on site.

(2) Mackenzie River Biomonitoring Project, 2005-2006: 102 sites (tributaries and mainstem) along the Mackenzie River from Great Slave Lake to the Delta were sampled by 
kick net (wadeable streams) and ponar grab (large rivers) in early fall (late August/early September) and preserved for later sorting as part of a biomonitoring programme led by Department of Fisheries and Oceans Canada scientist Laura Rempel, and Ephemeroptera specimens were made available for this study (Rempel and Gill 2010).

(3) Yukon streams, 2006: streams along the Klondike Highway near Whitehorse and in the Ogilvie Mountains along the Dempster Highway to the Yukon/Northwest Territories border were sampled by kick net and sorted on site in July 2006.

(4) Norman Wells biomonitoring study, 20102011: Samples were collected monthly between June and October by kick/dip net in streams and adjacent ponds near Norman Wells, supplemented by aerial collections and Malaise trapping, in cooperation with the Sahtu Renewable Resources Board (Vinke et al. 2015).

(5) Northern Biodiversity Programme, 20102011: samples were collected from aquatic habitats near Yellowknife, Norman Wells, and Banks Island and sorted on site. A subsample of these was submitted to the Biodiversity Institute of Ontario, University of Guelph (Guelph, Ontario, Canada) for DNA barcoding (Buddle et al. 2008; Cordero et al. 2016).

All specimens were collected into $80 \%$ ethanol, except for those collected for the barcoding study (preserved in 95\% ethanol) and by L. Rempel (collected into $10 \%$ formalin, then washed into $70 \%$ ethanol for processing). Approximately 1500 specimens from these recent collections were large and intact enough to identify at least to genus.

Working with archived samples: The archived samples from the 1970s Mackenzie River pipeline study were generally in good condition, though some were in discoloured ethanol due to bleeding from the rubber stopper, or contaminated with residual oil from oil spill experiments in the original study. A major challenge to working with these specimens was the interpretation of coded labels (Fig. 2) from the ecological study, which was a necessary first step to validating samples for biodiversity assessment. Labels in all the vials from this study contained at least a site/date/sample code (Fig. 2A), and only about $20 \%$ had labels with additional site or date information, though many of these were too degraded to read (Fig. 2B). Family determinations were generally written on the front or back of the labels, occasionally supplemented by a genus or species determination, often with the name and date of the person who identified the specimens in that vial (Fig. 2C). More frequently, specimens that could be identified to genus or species were isolated from the rest of the vial contents in micro-vials, and each microvial had its own determination label (Fig. 2C). There were usually multiple species or genera in individual vials. Information to decipher the coded labels was found in Brunskill et al. (1973), but was not clearly indicated, and required considerable searching through the 472-page document and consultation with the original researchers. The breakdown of the code is shown in Figure 2D.

Specimen processing: Once labels were decoded, vials were sorted and grouped by site, replicate, and date, and the vial contents were examined and identified. Specimens were identified to genus using Waltz and Burian (2008), updated with couplet "patches" to reflect new taxonomic treatments of various genera (e.g., Jacobus and Wiersema 2014). Specimens that were mature and intact enough to identify to species were separated, then identified by consulting current revisions for individual genera, and comparing characteristics to original species descriptions and identified and verified reference material held at the Northeast Ephemeroptera Laboratory Research Collection at the Department of Biology, Southern Connecticut State University (New Haven, Connecticut, United States of America). The abbreviation "cf." is used to indicate that the specimen(s) examined share many character states with the species named but may actually represent a different taxon.

Updated identifications from the Mackenzie River pipeline study were compared to the original label determinations, to published species lists for the project, and to mayflies collected since 2000 , to assess the benefits of re-examining the historic material in assessing regional biodiversity in the area.

\section{Results}

Despite the age of the archived specimens, many of the larger specimens were in good enough shape to identify, at least to genus. Taxa 
Fig. 2. Sample labels from Mackenzie pipeline study. A. Sample coded labels of the type found in most vials. B. Labels showing location and date information as well as the standard codes; many of these were badly degraded as shown in the label at the right. C. Samples of taxa determination labels, showing the determination on the back of a site label, on the front of the label with the code, and on tiny labels placed into micro-vials. D. Example of how labels were decoded using Brunskill et al. (1973).
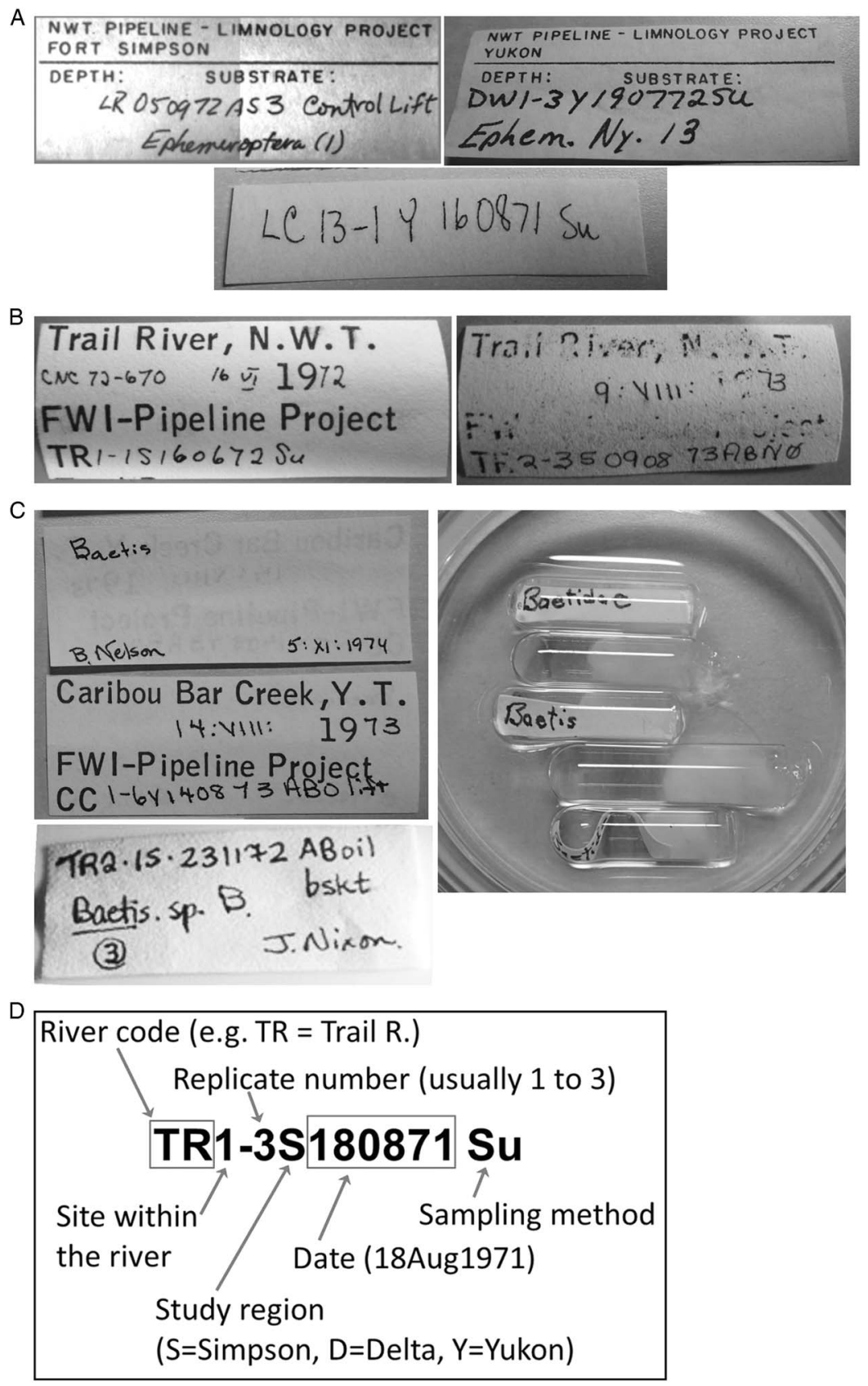

determination labels from the 1970 s that were in the vials generally matched up well with the taxa lists published in Wiens et al. (1975) and Cobb and Flannagan (1980), suggesting that the vials re-examined were a good representation of the original study material. 
Table 1. Total specimens that could be identified to family, genus, or species, compared between original vial labels and re-examined specimens.

\begin{tabular}{|c|c|c|c|c|c|c|c|c|c|c|c|}
\hline \multirow[b]{4}{*}{ Family } & \multirow[b]{4}{*}{ Total no. } & \multicolumn{6}{|c|}{ Data from pipeline labels } & \multirow{2}{*}{\multicolumn{4}{|c|}{ Data from updated labels }} \\
\hline & & \multicolumn{6}{|c|}{ Total individuals identified to this level } & & & & \\
\hline & & \multicolumn{2}{|c|}{ Family } & \multicolumn{2}{|c|}{ Genus } & \multicolumn{2}{|c|}{ Species } & \multicolumn{4}{|c|}{ Total individuals identified to this level } \\
\hline & & Corr & Inval & Corr & Inval & Corr & Inval & Family & Genus & Species & cf.sp. \\
\hline Ameletidae & 188 & 2 & 12 & 172 & 0 & 2 & 0 & 0 & 91 & 8 & 89 \\
\hline Ametropodidae & 1 & 0 & 1 & 0 & 0 & 0 & 0 & 0 & 0 & 1 & 0 \\
\hline Baetidae & 8959 & 7082 & 10 & 1208 & 659 & 0 & 0 & 5520 & 2038 & 1203 & 198 \\
\hline Baetiscidae & 10 & 0 & 0 & 10 & 0 & 0 & 0 & 0 & 0 & 10 & 0 \\
\hline Caenidae & 282 & 78 & 1 & 203 & 0 & 0 & 0 & 0 & 0 & 282 & 0 \\
\hline Ephemerellidae & 581 & 165 & 6 & 286 & 2 & 37 & 85 & 173 & 153 & 201 & 54 \\
\hline Ephemeridae & 15 & 3 & 0 & 11 & 0 & 1 & 0 & 0 & 3 & 11 & 1 \\
\hline Heptageniidae & 1868 & 970 & 12 & 672 & 164 & 12 & 38 & 497 & 650 & 353 & 368 \\
\hline Leptophlebiidae & 161 & 36 & 0 & 122 & 3 & 0 & 0 & 2 & 68 & 88 & 3 \\
\hline Metretopodidae & 95 & 17 & 4 & 74 & 0 & 0 & 0 & 0 & 1 & 91 & 3 \\
\hline Siphlonuridae & 55 & 0 & 7 & 47 & 0 & 0 & 1 & 20 & 11 & 24 & 0 \\
\hline Total & 12215 & 8353 & 53 & 2805 & 828 & 52 & 124 & 6212 & 3015 & 2272 & 716 \\
\hline
\end{tabular}

Note: For "pipeline labels", numbers indicate whether the identification on the old labels was still correct or current (Corr) or were misidentified, represented names that have been synonymised, superseded, or were otherwise invalid, or where no determination label was present (Inval) (see details within each family). "cf.sp." refers to tentative species identifications. Where the only change was a subgenus name that has been elevated to genus (but was otherwise correct), label identification was considered to be correct (e.g., Ephemerella bicolor $=$ Eurylophella bicolor, Heptagenia hebe $=$ Leucrocuta hebe, and Stenonema vicarium $=$ Maccaffertium vicarium).

Nearly $70 \%$ of the specimens (8353 of 12215 ) were identified only to the family level in the 1970s study (Table 1). Many of these were very small, so even with improved taxonomic resources, it was still not possible to identify the majority of them (6212 specimens) further than family. However, an additional 2141 specimens (a further 18\%) could be identified at least to genus in the current study (Table 1), thanks to the availability of updated taxonomic resources (keys and revisionary works) and improved understanding of how the older taxonomic literature related to the current species characteristics. In the 1970s study, only 176 specimens were identified to species (52 of which are still current and were correctly identified; Table 1), resulting in a list of 17 species (six of which are still correct) (Tables 2-3). In the current study, 2272 specimens were identifiable to species, giving 45 species in total. When these were added to specimens from our "new" (post-2000) sampling in the region, 74 species of mayfly were identified from Yukon and the Northwest Territories (Tables 2, 3).

\section{Comparing original 1970s pipeline study label data with updated identifications}

Genus-level identifications: A total of 3633 specimens were originally identified to genus, and of these, a little over half were correctly placed into a genus that is still valid taxonomically (including those that could be further identified to species) (Tables 1,3). All or most of the specimens identified to genus were found to still be correctly assigned to that genus in the families Ameletidae, Baetiscidae, Caenidae, Ephemeridae, Metretopodidae, and Siphlonuridae (see Table 3 for details within each family). However, taxonomic revisions in the Baetidae, Ephemerellidae, and Heptageniidae resulted in many of the specimens formerly placed in large "catch-all" genera (like Baetis, Ephemerella Walsh, and Heptagenia Walsh) to now be placed in new or different genera (Table 3). This was particularly true in the Baetidae, the family that made up nearly three-quarters of the mayflies examined.

For Baetidae and Heptageniidae, it was necessary to physically examine specimens to update the list of genera, since genus names used in the 
Table 2. Numbers of genera and species listed on original pipeline study labels (Yukon and Northwest Territories combined) compared to those resulting from the re-examination of the specimens and new collections for this study.

\begin{tabular}{|c|c|c|c|c|c|c|c|c|c|c|c|c|c|}
\hline \multirow[b]{3}{*}{ Family } & \multicolumn{6}{|c|}{$\begin{array}{l}\text { Data from pipeline labels } \\
\text { Total number of genera or species listed }\end{array}$} & \multirow{2}{*}{\multicolumn{3}{|c|}{$\begin{array}{c}\text { Data from re-examination } \\
\text { Total number of genera or species }\end{array}$}} & \multirow{2}{*}{\multicolumn{2}{|c|}{$\begin{array}{l}\text { Recent collections } \\
\text { (including previous “cf.") }\end{array}$}} & \multirow{2}{*}{\multicolumn{2}{|c|}{ Overall total }} \\
\hline & \multicolumn{3}{|c|}{ Genera } & \multicolumn{3}{|c|}{ Species } & & & & & & & \\
\hline & Total listed & Invalid & Valid & Total listed & Invalid & Valid & Genus & Species & Extra cf.sp. & Extra genus & Extra species & Species & cf.sp. \\
\hline Ameletidae & 1 & 0 & $\mathbf{1}$ & 1 & 1 & $\mathbf{0}$ & 1 & 1 & 3 & 0 & 4 & 5 & 1 \\
\hline Ametropodidae & 0 & 0 & $\mathbf{0}$ & 0 & 0 & $\mathbf{0}$ & 1 & 1 & 0 & 0 & $\mathbf{0}$ & 1 & 0 \\
\hline Baetidae & 4 & 3 & 1 & 0 & 0 & $\mathbf{0}$ & 5 & 14 & 3 & 3 & 8 & 22 & 2 \\
\hline Baetiscidae & 1 & 0 & 1 & 0 & 0 & $\mathbf{0}$ & 1 & 1 & 0 & 0 & $\mathbf{0}$ & 1 & 0 \\
\hline Caenidae & 1 & 0 & 1 & 0 & 0 & $\mathbf{0}$ & 1 & 4 & 0 & 0 & $\mathbf{0}$ & 4 & 0 \\
\hline Ephemerellidae & 7 & 5 & 2 & 7 & 5 & 2 & 5 & 6 & 2 & 1 & 6 & 12 & 3 \\
\hline Ephemeridae & 1 & 0 & 1 & 1 & 0 & 1 & 1 & 1 & 0 & 0 & $\mathbf{0}$ & 1 & 0 \\
\hline Heptageniidae & 5 & 2 & 3 & 7 & 5 & 2 & 8 & 10 & 4 & 3 & 10 & 20 & 5 \\
\hline Leptophlebiidae & 1 & 0 & 1 & 0 & 0 & $\mathbf{0}$ & 2 & 3 & 0 & 0 & $\mathbf{0}$ & 3 & 0 \\
\hline Metretopodidae & 2 & 0 & 2 & 0 & 0 & $\mathbf{0}$ & 2 & 2 & 1 & 0 & $\mathbf{0}$ & 2 & 1 \\
\hline Siphlonuridae & 2 & 0 & 2 & 1 & 1 & $\mathbf{0}$ & 2 & 2 & 0 & 0 & 1 & 3 & 0 \\
\hline Total & 25 & 10 & 15 & 17 & 11 & 6 & 29 & 45 & 12 & 7 & 29 & 74 & 12 \\
\hline
\end{tabular}

Note: "Pipeline label" data columns indicate total listed as well as those found to be incorrect or represented names that have been synonymised, superseded, or otherwise invalid (Inval) (Table 1 for details within each family). The "cf.sp." columns refer to tentative species. Numbers in bold text represent total valid species. 
Table 3. Comparison of taxa lists generated from the original label data from Mackenzie Valley pipeline vials, the updated information following re-examination of specimens, and new (post-2000) sampling in Yukon and Northwest Territories, Canada (see Fig. 1 for collecting localities).

Total number of specimens

\begin{tabular}{|c|c|c|c|c|c|c|c|c|}
\hline & \multicolumn{6}{|c|}{ Total number of specimens } & & \\
\hline & \multicolumn{2}{|c|}{$\begin{array}{l}\text { Original pipeline study } \\
\text { label data }\end{array}$} & \multicolumn{2}{|c|}{$\begin{array}{l}\text { Updated pipeline } \\
\text { study data }\end{array}$} & \multicolumn{2}{|c|}{ Data from new sampling } & \multicolumn{2}{|c|}{ Literature records } \\
\hline & YK & NWT & YK & NWT & YK & NWT & YK & NWT \\
\hline Ameletidae (total: 188) & & 2 & & & & & & \\
\hline No determination label; updated to Ameletus & & 1 & & & & & - & - \\
\hline Mislabelled as Baetidae; corrected to Ameletus & 4 & 7 & & & & & - & - \\
\hline $\begin{array}{l}\text { Previously identified as Ameletus celeroides }(=\text { A. celer }) \text {; too small and } \\
\text { faded to identify; now Ameletus species }\end{array}$ & & 2 & & & & & - & - \\
\hline Ameletus celer McDunnough & & & & & $\mathrm{x}$ & & 4 & - \\
\hline $\begin{array}{l}\text { Ameletus species (most of original ones labelled Ameletus still identified as } \\
\text { Ameletus, except those listed below) }\end{array}$ & 8 & 164 & 4 & 87 & & 15 & - & - \\
\hline Ameletus cooki McDunnough "cf." & & & & 1 & 2 & & NR? & NR? \\
\hline Ameletus inopinatus Eaton & & & & & $\mathrm{x}$ & 8 & 1,4 & $1,4,10,12$ \\
\hline Ameletus inopinatus Eaton "cf." & & & 8 & & & & - & - \\
\hline Ameletus oregonensis McDunnough & & & & & & 2 & - & 14 \\
\hline Ameletus similior McDunnough? & & & & & & 1 & - & NR? \\
\hline Ameletus subnotatus Eaton & & & & 8 & & & - & NR \\
\hline Ameletus ubnotatus Eaton "cf." & & & & 80 & & & - & - \\
\hline Ameletus velox Dodds & & & & & $\mathrm{x}$ & & 4 & - \\
\hline Ametropodidae (total: 1 ) & & & & & & & _- & _ \\
\hline No determination label; updated to Ametropus fragilis & & 1 & & & & & - & - \\
\hline Ametropus fragilis Albarda & & & & 1 & & & - & $1,2,10$ \\
\hline Baetidae (total: 8959) (many of these were large enough to identify to at least genus) & 3097 & 3985 & 2911 & 2609 & & & - & - \\
\hline No determination label; updated to Acentrella turbida & & 2 & & & & & - & - \\
\hline Previously identified as Pseudocloeon; updated to different & 95 & 329 & & & & & - & - \\
\hline $\begin{array}{l}\text { Acentrella species (131), Acerpenna pygmaea (9); Plauditus } \\
\text { species (92); Baetidae (192) }\end{array}$ & & & & & & & & \\
\hline Previously identified as Cloeon; corrected to Procloen & & 1 & & & & & - & - \\
\hline $\begin{array}{l}\text { Previously identified as Centroptilum; corrected to Acentrella } \\
\text { lapponica; (5); Baetis flavistriga (80); Baetis bundyae (41) }\end{array}$ & 126 & & & & & & - & - \\
\hline Previously identified as Baetinae corrected to Baetis flavistriga & 15 & & & & & & - & - \\
\hline Mislabelled as Heptageniids; corrected to different Acentrella species & 10 & & & & & & - & - \\
\hline Acentrella species & & & 23 & 2 & & 1 & - & - \\
\hline Acentrella feropagus Alba-Tercedor and McCafferty & & & 2 & & & & 1,10 & _- \\
\hline Acentrella feropagus Alba-Tercedor and McCafferty "cf." & & & & & & 1 & - & - \\
\hline Acentrella insignificans (McDunnough) & & & 1 & 16 & & 13 & $1,8,10$ & 1,10 \\
\hline Acentrella insignificans (McDunnough) "cf." & & & & 1 & & & - & 1 \\
\hline Acentrella lapponica Bengtsson & & & 150 & & & 52 & $\overline{1}$ & 1,4 \\
\hline
\end{tabular}


Acentrella lapponica Bengtsson "cf."

Acentrella turbida (McDunnough)

Acentrella turbida (McDunnough) "cf."

Acentrella new species

Acerpenna species

Acerpenna pygmaea (Hagen)

Acerpenna pygmaea (Hagen) "cf."

Anafroptilum species

Anafroptilum album (McDunnough)

Baetis species (many of the specimens previously identified to Baetis have been updated to different species of Acentrella,

Acerpenna, Plauditus, and Procloeon)

Baetis bicaudatus Dodds

Baetis bicaudatus Dodds "cf."

Baetis brunneicolor McDunnough

Baetis brunneicolor McDunnough "cf."

Baetis bundyae Lehmkuhl

Baetis flavistriga McDunnough (species complex)

Baetis flavistriga McDunnough "cf."

Baetis foemina McDunnough

Baetis foemina McDunnough "cf."

Baetis hudsonicus Ide

Baetis tricaudatus Dodds

Baetis tricaudatus Dodds "cf."

Baetis vernus Curtis

Baetis vernus Curtis ("cf." or new species)

Callibaetis ferrugineus Hagen

Diphetor hageni (Eaton)

Plauditus species

Plauditus cestus (Provonsha and McCafferty)

Plauditus dubius (Walsh)

Plauditus gloveri McCafferty and Waltz

Plauditus gloveri McCafferty and Waltz "cf."

Plauditus punctiventris (McDunnough) "cf."

Plauditus virilis (McDunnough)

Procloeon species
Total number of specimens

\begin{tabular}{|c|c|c|c|c|c|c|c|}
\hline \multicolumn{2}{|c|}{$\begin{array}{l}\text { Original pipeline study } \\
\text { label data }\end{array}$} & \multicolumn{2}{|c|}{$\begin{array}{l}\text { Updated pipeline } \\
\text { study data }\end{array}$} & \multicolumn{2}{|c|}{ Data from new sampling } & \multicolumn{2}{|c|}{ Literature records } \\
\hline YK & NWT & YK & NWT & YK & NWT & YK & NWT \\
\hline & & 1 & & & 1 & 1 & 1 \\
\hline & & 48 & 29 & & 38 & 13 & 14 \\
\hline & & 5 & & & 18 & - & - \\
\hline & & 32 & & & & NR & - \\
\hline & & & 1723 & & & - & - \\
\hline & & & 40 & & & NR & 14 \\
\hline & & & 3 & 1 & 1 & $\overline{3}$ & $\overline{3,11}$ \\
\hline & & & & 1 & 9 & _- & 14 \\
\hline \multirow[t]{25}{*}{379} & 920 & 18 & 238 & & 18 & - & - \\
\hline & & 12 & 8 & 10 & 105 & $1,2,4,8,10,11$ & $1,2,10,14$ \\
\hline & & & 9 & & 10 & - & - \\
\hline & & 34 & 37 & 25 & 28 & NR & NR \\
\hline & & 2 & & & & - & - \\
\hline & & 42 & & & 103 & $1,8,10$ & $1,7,10$ \\
\hline & & 382 & 95 & 1 & 37 & 1,13 & $1,10,14$ \\
\hline & & & 3 & & & - & - \\
\hline & & & & & 34 & - & $1,3,9$ \\
\hline & & & & & 2 & - & - \\
\hline & & & & & $\mathrm{x}$ & - & $\begin{array}{c}1,4 \\
1,2,3,4,9,10,14\end{array}$ \\
\hline & & 45 & 198 & 18 & 129 & $1,2,8,10$ & $1,2,3,4,9,10,14$ \\
\hline & & 5 & 83 & 1 & 6 & - & - \\
\hline & & & & & $\mathrm{x}$ & - & 4 \\
\hline & & 7 & & & 13 & NR? & - \\
\hline & & & & & 1 & $1,7,8,10$ & $1,2,10$ \\
\hline & & & & 2 & & NR & 1,10 \\
\hline & & & 26 & & 3 & - & - \\
\hline & & & & & 2 & - & $1,6,14$ \\
\hline & & & 1 & & & - & NR \\
\hline & & & 2 & & & - & NR \\
\hline & & & 77 & & & - & - \\
\hline & & & 2 & & & - & NR? \\
\hline & & & 1 & & & - & NR \\
\hline & & 1 & 5 & & & - & - \\
\hline
\end{tabular}


Total number of specimens

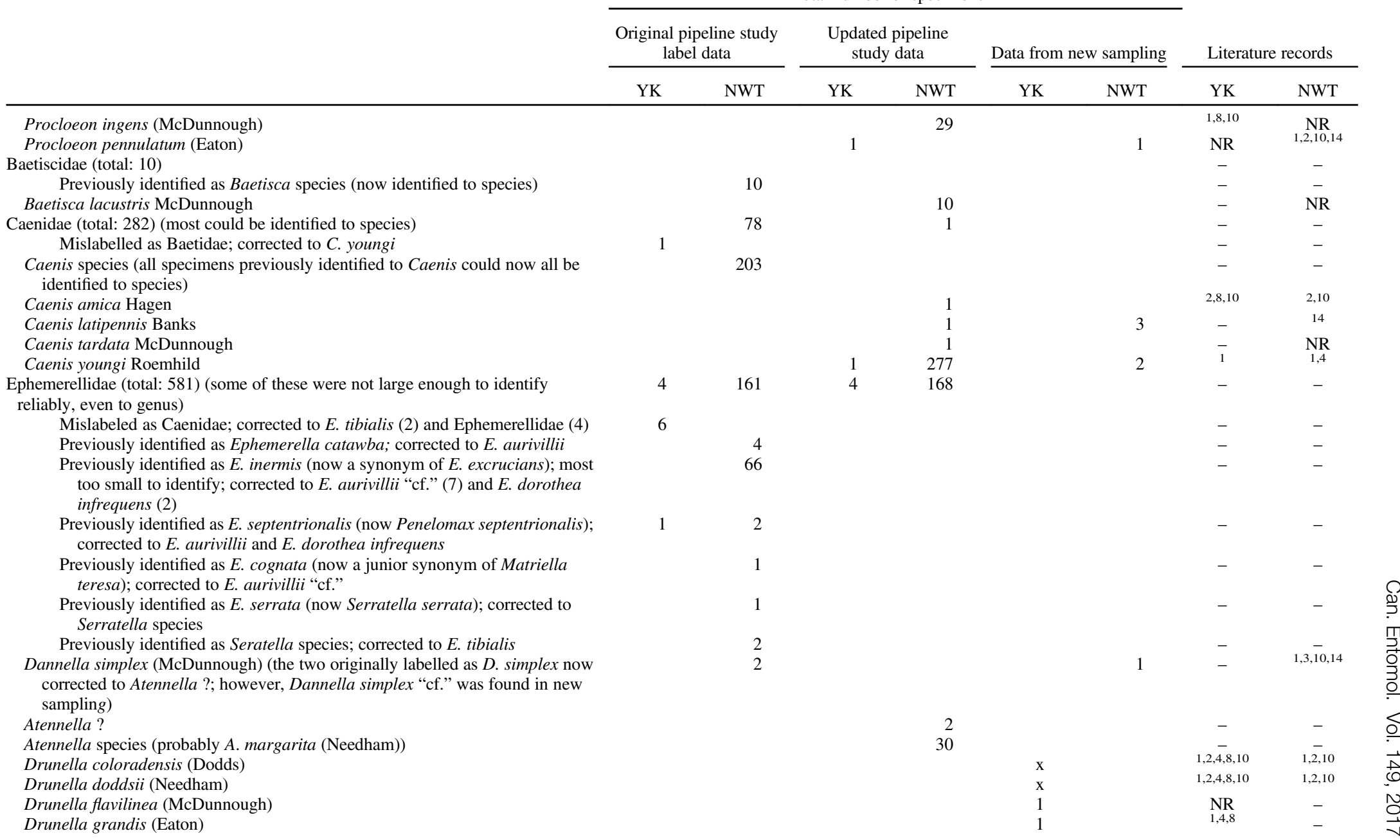


Ephemerella species (many of the specimens originally identified as Ephemerella species were large enough to identify to species, and are listed below)

Ephemerella aurivillii (Bengtsson) (the 9 specimens originally identified as

E. aurivillii corrected to E. mucronata)

Ephemerella aurivillii (Bengtsson) "cf."

Ephemerella dorothea infrequens McDunnough

Ephemerella dorothea infrequens McDunnough "cf."

Ephemerella excrucians Walsh

Ephemerella mucronata (Bengtsson)

Ephemerella cf. mucronata Bengtsson "cf."

Ephemerella (Vittapalia) tibialis McDunnough

Eurylophella species

Eurylophella bicolor (Clemens); originally identified as Ephemerella bicolor updated to Eurylophella

Updated pipeline

\begin{tabular}{|c|c|c|c|c|c|c|c|}
\hline \multicolumn{2}{|c|}{$\begin{array}{c}\text { Original pipeline study } \\
\text { label data }\end{array}$} & \multicolumn{2}{|c|}{$\begin{array}{l}\text { Updated pipeline } \\
\text { study data }\end{array}$} & \multicolumn{2}{|c|}{ Data from new sampling } & \multicolumn{2}{|c|}{ Literature records } \\
\hline YK & NWT & YK & NWT & YK & NWT & YK & NWT \\
\hline
\end{tabular}

53

233

YK

95

NWT

YK

Eurylophella temporalis (McDunnough)

Serratella species (two originally labelled as Serratella species were corrected to

E. tibialis; the updated Serratella listed here was previously identified as

$$
\text { E. serrata }
$$

Ephemeridae (total: 15)

Ephemera species

Ephemera simulans Walker

Ephemera simulans Walker "cf."

Heptageniidae (total: 1868) (many of these were large and intact enough to identify to at least genus; see list below)

No determination on label; updated to Leucrocuta ?

Mislabelled as Callibaetis; (corrected to Cinygmula species (subimago))

Mislabelled as Baetidae; (corrected to Cinygmula species)

Mislabelled as Metretopus; corrected to Leucrocuta "cf."

Epeorus (5); corrected to Acentrella (listed above under Acentrella)

Heptagenia julia; corrected to Heptagenia species (1); Heptagenia

solitaria "cf." (4)

Heptagenia hebe or H. maculipennis; updated to Leucrocuta hebe (listed below)

Stenacron pallidum; corrected to Heptagenia species

Stenonema species; updated to Maccaffertium species

Stenonema birdi (now synonym of Stenonema femoratum); corrected to

Stenonema luteum

Stenonema pulchellum; corrected to Stenonema terminatum

9

10

23

29

8

40

1
2

43

37

16
4

86

14

\begin{tabular}{|c|c|c|}
\hline 23 & - & $1,3,4,7,9,10,14$ \\
\hline 10 & - & - \\
\hline \multirow[t]{4}{*}{93} & NR & 14 \\
\hline & - & $\frac{-}{123.1011}$ \\
\hline & $\overline{1,7,8,10}$ & $1,7,10$ \\
\hline & - & - \\
\hline \multirow[t]{3}{*}{1} & NR & $1,2,10$ \\
\hline & - & - \\
\hline & - & 1,3 \\
\hline \multirow[t]{2}{*}{1} & - & $1,4,5,10$ \\
\hline & - & - \\
\hline
\end{tabular}

3

11
1

470

325

3

11
172

172 
Total number of specimens

\begin{tabular}{|c|c|c|c|c|c|c|c|c|}
\hline & \multicolumn{6}{|c|}{ lotal number of specimens } & \multirow{2}{*}{\multicolumn{2}{|c|}{ Literature records }} \\
\hline & \multicolumn{2}{|c|}{$\begin{array}{l}\text { Original pipeline study } \\
\text { label data }\end{array}$} & \multicolumn{2}{|c|}{$\begin{array}{l}\text { Updated pipeline } \\
\text { study data }\end{array}$} & \multicolumn{2}{|c|}{ Data from new sampling } & & \\
\hline & YK & NWT & YK & NWT & YK & NWT & YK & NWT \\
\hline Anepeorus rusticus McDunnough (previously identified as Rhithrogena) & & & & 1 & & & - & NR \\
\hline $\begin{array}{l}\text { Cinymula species (some previously identified as Cinygmula species are now listed } \\
\text { under individual species below) }\end{array}$ & 129 & & 99 & & 21 & 20 & - & - \\
\hline $\begin{array}{l}\text { Cinymula species group A nymphs (lack filaments at base of abdominal gills } \\
\text { (group includes } C \text {. par, C. tarda, C. uniformis, and C. subaequalis)) }\end{array}$ & & & 221 & & & 35 & - & - \\
\hline $\begin{array}{l}\text { Cinymula species group B nymphs (have filaments at base of abdominal gills } \\
\text { (group includes C. mimus, C. ramaleyi, } C \text {. gartrelli, } \text { C. kootenai, and } \\
\text { C. reticulata)) }\end{array}$ & & & & & & 8 & - & - \\
\hline Cinygmula mimus (Eaton) (adult specimens) & & & & & & 2 & $1,8,10$ & NR \\
\hline Cinygmula par (Eaton) (adult specimen) & & & & & & 1 & $1,8,10$ & NR \\
\hline Cinygmula subaequalis (Banks) & & & & & $\mathrm{x}$ & $\mathrm{x}$ & 4 & 4 \\
\hline Cinymula tarda McDunnough (adult specimens) & & & & & & $\hat{2}$ & 1 & $1,3,10$ \\
\hline Ecdyonurus simplicioides (McDunnough) "cf." & & & 1 & & & & NR? & - \\
\hline Epeorus albertae (McDunnough) & & & & & & 1 & - & NR \\
\hline Epeorus albertae (McDunnough) “cf." & & & & & 3 & & NR? & - \\
\hline Epeorus deceptivus (McDunnough) "cf." & & & & & & 1 & - & $1,3,9,10$ \\
\hline Epeorus grandis (McDunnough) & & & & & $\mathrm{x}$ & & $1,8,4,10$ & $1,2,10$ \\
\hline Epeorus longimanus (Eaton) & & & & & $\begin{array}{l}\Lambda \\
9\end{array}$ & & $1,8,10$ & $1,2,10$ \\
\hline $\begin{array}{l}\text { Heptagenia species (some previously identified as Heptagenia species are now } \\
\text { listed under individual species below) }\end{array}$ & 27 & 505 & 5 & 78 & & 56 & - & - \\
\hline Heptagenia elegantula (Eaton) & & & & 168 & & & - & NR \\
\hline Heptagenia elegantula (Eaton) "cf." & & & & & & 1 & - & - \\
\hline Heptagenia pulla (Clemens) (western form) & & & & 112 & & 3 & $1,2,8,10$ & $1,3,4,7,9,10,11$ \\
\hline Heptagenia solitaria McDunnough & & & & 9 & & 30 & - & 1,14 \\
\hline Heptagenia solitaria McDunnough "cf." & & & & 337 & & 43 & - & - \\
\hline Leucrocuta species & & & & 30 & & & - & - \\
\hline Leucrocuta hebe (McDunnough) & & & & 3 & & & - & $1,3,10,11$ \\
\hline Maccaffertium species & & & & 57 & & & - & - \\
\hline Maccaffertium luteum (Clemens) & & & & 3 & & & - & NR \\
\hline Maccaffertium luteum (Clemens) "cf." & & & & 17 & & & - & - \\
\hline Maccaffertium terminatum terminatum (Walsh) & & & & 9 & & & - & NR \\
\hline $\begin{array}{l}\text { Maccaffertium vicarium (Walker) (original ones identified as Stenonema vicarium } \\
\text { and are now updated to Maccaffertium vicarium) }\end{array}$ & & 17 & & 40 & & & - & $1,3,10$ \\
\hline Nixe species & & & 1 & 3 & & & - & - \\
\hline Nixe lucidipennis (Clemens) & & & 6 & & & & NR & - \\
\hline Nixe lucidipennis (Clemens) "cf." & & & 1 & & & & - & - \\
\hline
\end{tabular}


Total number of specimens

\begin{tabular}{|c|c|c|c|c|c|c|c|c|}
\hline & & \multirow{2}{*}{\multicolumn{2}{|c|}{ Literature records }} \\
\hline & & $\begin{array}{c}\text { Original pipeline study } \\
\text { label data }\end{array}$ & $\begin{array}{r}\text { Upda } \\
\text { st }\end{array}$ & $\begin{array}{l}\text { peline } \\
\text { ata }\end{array}$ & Data fro & sampling & & \\
\hline & & NWT & YK & NWT & YK & NWT & YK & NWT \\
\hline
\end{tabular}

Nixe rusticalis (McDunnough)

Rhithrogena species (some of these originally identified as Rhithrogena species

48

61

57

99

128

could be tentatively identified to species, see list below. Some others now

included in Rhithrogena were previously misidentified in other genera)

Rhithrogena hageni Eaton "cf."

Rhithrogena jejuna Eaton

Rhithrogena morrisoni (Banks) "cf."

Rhithrogena robusta Dodds "cf."

Rhithrogena undulata (Banks) "cf."

Stenacron interpunctatum (Say)

Stenonema femoratum (Say)

Leptophlebiidae (total: 161) (most of these could be identified at least to genus)

Identified as Leptophlebiinae; now updated to Paraleptophlebia debilis

Leptophlebia species (many of these originally identified as

Leptophlebia species could now be identified to species)

Leptophlebia cupida (Say)

Leptophlebia cupida (Say) "cf."

Leptophlebia nebulosa (Walker)

Leptophlebia nebulosa (Walker) "cf."

Paraleptophlebia species

Paraleptophlebia debilis (Walker)

Paraleptophlebia debilis (Walker) "cf."

Metretopodidae (total 95)

Mislabelled as Baetidae, updated to Metretopus borealis "cf."

Mislabelled as Siphlonuridae, updated to Metretopus borealis

Mislabelled as Siphlonurus, updated to Metretopus borealis

Metretopus borealis (Eaton)

Metretopus borealis (Eaton) "cf."

Siphloplecton species

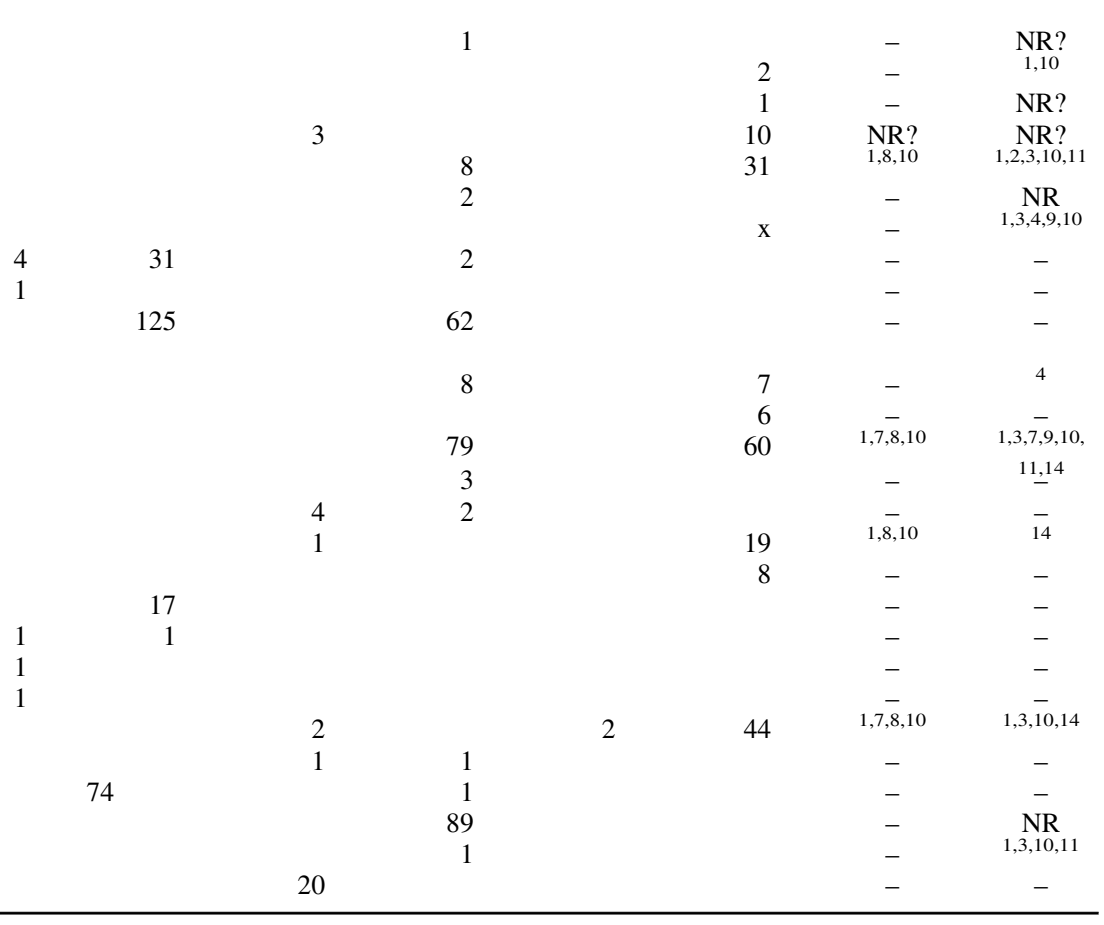

Siphloplecton basale Walker

Siphloplecton interlineatum (Walsh) "cf."

Siphlonuridae: (total 55) 
Total number of specimens

\begin{tabular}{|c|c|c|c|c|c|c|c|c|}
\hline & \multicolumn{6}{|c|}{ Total number of specimens } & & \\
\hline & \multicolumn{2}{|c|}{$\begin{array}{l}\text { Original pipeline study } \\
\text { label data }\end{array}$} & \multicolumn{2}{|c|}{$\begin{array}{l}\text { Updated pipeline } \\
\text { study data }\end{array}$} & \multicolumn{2}{|c|}{ Data from new sampling } & \multicolumn{2}{|c|}{ Literature records } \\
\hline & YK & NWT & YK & NWT & YK & NWT & YK & NWT \\
\hline $\begin{array}{l}\text { Mislabelled as Ameletus; updated to Parameletus species (1) and Siphlonurus } \\
\text { species (4) }\end{array}$ & 5 & & & & & & - & - \\
\hline Mislabelled as Cloeon (subimago), updated to Siphlonurus species & 2 & & & & & & - & - \\
\hline Parameletus species & & 24 & 1 & 1 & & 1 & - & - \\
\hline Parameletus chelifer Bengtsson1 & & & & 23 & & 60 & - & $1,4,7,10$ \\
\hline Siphlonurus species & 22 & 1 & 8 & 1 & & 2 & - & - \\
\hline $\begin{array}{l}\text { Siphlonurus occidentalis Eaton (specimen originally identified as } S \text {. occidentalis } \\
\text { corrected to } S \text {. alternatus. However, S. occidentalis was collected in recent } \\
\text { sampling) }\end{array}$ & & 1 & & & & 1 & $1,2,7,8,10$ & $1,2,10,11$ \\
\hline Siphlonurus alternatus (Say) & & & & 1 & & 6 & 1,8 & $1,7,11$ \\
\hline Total specimens & 4541 & 7674 & 4541 & 7674 & 111 & 1384 & & \\
\hline Total number of named species (by territory) & 1 & 17 & 14 & 39 & 17 & 41 & & \\
\hline Total number of named species (by study) & 17 & & 46 & & 50 & & & \\
\hline Total number named species (updated) & & & & & 25 & 68 & & \\
\hline Overall total (YK + NT, updated + new sampling) & & & & & 74 & & & \\
\hline
\end{tabular}

Notes: Total number of specimens is given for all collections except Cordero et al. 2016, where "x" denotes presence based on barcode analysis. Deeply indented entries in the first column indicate misidentifications or invalid names from the original pipeline study labels and how they were corrected. Literature records: ${ }^{1} \mathrm{Mayfly}$ Central (2016), given as “Canada far north"; ${ }^{2} \mathrm{Cobb}$ et al. (1995); ${ }^{3}$ Cobb and Flannagan (1980); ${ }^{4}$ Cordero et al. (2016); ${ }^{5}$ Funk and Sweeney (1994); ${ }^{6}$ Gorski et al. (2014); ${ }^{7}$ Harper and Harper (1981); ${ }^{8}$ Harper and Harper (1997); ${ }^{7}$ Moore (1977); ${ }^{10}$ Randolph (2002); ${ }^{11}$ Wiens et al. (1975); ${ }^{12}$ Zloty (1996); ${ }^{13}$ McCafferty et al. (2004); ${ }^{14}$ Vinke et al. (2015).

Territorial records: NR, new record; NR?, potential new record, but species identification is tentative only. YK, Yukon; NWT, Northwest Territories.

Identifications: The abbreviation "cf." is used to indicate that the specimen(s) examined share many character states with the species named but may actually represent a different taxon. A species or genus name followed by a question mark (?) represents a tentative identification where specimens were too small for positive identification, but the identification is likely correct. 
1970s survey could not be used to predict the groupings of genera identified using updated taxonomic resources. This was particularly true for the Baetidae, as specimens did not fit into discrete new genera derived from previously named genera. For example, specimens originally labelled "Pseudocloeon sp." (a genus no longer recognised in North America) were re-identified as species of Acentrella Bengtsson, Acerpenna Waltz and McCafferty, or Plauditus Lugo-Ortiz and McCafferty. Similarly, those previously identified as "Centroptilum sp." were updated as species in Acentrella or Baetis Leach, and those previously identified as "Baetis sp." were identified as species in Acentrella, Acerpenna, Baetis, Plauditus, or Procloeon Bengtsson. However, there have been no changes in genera for groups such as Ameletus Eaton or Caenis Stephens, so these showed no change between the 1970s and the current study.

Species-level identifications: Of the 176 specimens originally identified to species (based on presence of species determination labels in the vials), only 52 of these still had current names or were identified correctly (Tables 1, 3). This compares to 2272 that could be identified to species in the current study. However, the success of updated species identification varied widely by family, depending on both the state and size of the specimens and on the availability of updated taxonomic resources. All, or nearly all, the specimens in Ametropodidae, Baetiscidae, Caenidae, and Metretopodidae could be now be identified to species, about three-quarters of the Ephemeridae could be identified to species, and about half of the Leptophlebiidae and Siphlonuridae could be identified to species. In contrast, only 4, 13, and $19 \%$ of ameletid, baetid, and heptageniid specimens (respectively) could be identified to species after re-examination, though two of these were also the families that produced the greatest number of additional species during re-examination: 14 baetid species and 10 heptageniid species. The low percentage "success" rate in these groups was mainly due to the small size of the specimens: the original study collected bulk samples from many rivers over multiple years and seasons, giving a variety of size classes of specimens. The old specimens were also very faded, which made it difficult to identify groups where colour patterns were important characters for identification, such as the Ameletidae.

\section{Comparison with "new" sampling expeditions to Yukon and Northwest Territories}

Nearly 1500 specimens from the five additional sampling expeditions that included parts of Yukon and the Northwest Territories since 2000 were also examined and identified. These had the advantage of being newer, showing clear colour patterns and being morphologically intact, and in the case of the Northern Biodiversity Programme specimens, preserved specifically to allow DNA barcoding. Despite the relatively low numbers of specimens compared to the Mackenzie Valley pipeline study samples (1495 compared with 12215), the number of species in Yukon and Northwest Territories identified from the two sets of samples were similar (45 in the pipeline samples compared to 50 in the newly collected samples) (Table 3 ). There was some overlap in species composition, so the newer sampling added 29 additional species when compared to the pipeline study samples. When the lists were combined, the total climbed to 74 species (plus several tentative, "cf." identifications).

\section{Discussion}

The clear benefits in updating and clarifying previously published species lists from re-examining archived Mackenzie Valley pipeline study samples fully justified the challenges encountered in dealing with the samples. It was difficult and time consuming to locate the sample vials associated with this study and gather them into a single location so that the study could proceed, and it is likely that some were missed in the storage areas at the Freshwater Institute (Winnipeg) and the Canadian National Collection of Insects (Ottawa). Some vials had dried out, and could not be rehydrated. Further, lack of clear information on original specimen labels (including those deposited at the Canadian National Collection of Insects) was a major drawback to implementing this study, and required considerable research to resolve. However, the archived samples gave access to specimens over a vast geographical area from southern Northwest Territories $\left(\sim 60^{\circ} \mathrm{N}\right)$ to the Mackenzie Delta (to $68.5^{\circ} \mathrm{N}$ ) and west to northern Yukon (Brunskill et al. 1973), and over a variety of seasons and habitats that would be difficult and very expensive to sample today. Another major benefit 
was that samples were already sorted from the sample debris, resulting in a major cost savings in processing samples.

Re-examining the archived samples provided new data on relative abundance and distributional data for 45 species, several of which were not previously reported in published accounts for Yukon (seven species, plus two tentative (cf.) species) or the Northwest Territories (14 species plus three tentative (cf.) species; these are in addition to new records from this study already published in Vinke et al. 2015; Cordero et al. 2016). Nearly all of these required re-examination of original specimens to detect. An example of updated distributional data includes the records from river sites for Ameletus inopinatus Eaton (Ephemeroptera: Ameletidae) (previously identified as "Ameletus sp."). These expand the previously restricted habitat association in northern Canada (i.e., lakes and ponds, Zloty 1996) to habitats similar to Palaearctic populations (running waters in addition to lakes and ponds). In another example, a single specimen of Ametropus fragilis Albarda (Ephemeroptera: Ametropodidae), a rare, large river mayfly that was not even identified to family on original labels, was collected from the Mackenzie River just west of Inuvik. This species is reported to be endangered in all or part of its range (Jacobus 2013), so may be of conservation interest. Cobb and Flannagan (1980) reported this species in the Nahanni River in southern Northwest Territories, but this study shows that its distribution extends far to the north in the Mackenzie River Delta. Another rare mayfly, Anepeorus rusticus McDunnough (Ephemeroptera: Heptageniidae) (previously identified as "Rhithrogena sp." on pipeline study labels), had not been reported in the Northwest Territories before a single specimen was found from the Liard River in the pipeline samples. Although this is an old record, it demonstrates that greater effort should be made to specifically sample for it in the future to determine its distribution. Two fairly common heptageniid mayflies (Heptagenia pulla (Clemens) and Heptagenia solitaria McDunnough) also point to the importance of examining original specimens to assess species patterns. Hepagenia pulla shows two morphological forms in northern Canada, and the one in the study area covered by the pipeline study is the "western form", in which the asymmetrical mandibles are reversed compared with the eastern form; this may actually be a synonym of the Palaearctic species $H$. dalecarlica Bengtsson (Jacobus et al. 2014). Based on samples examined from across Nunavut and the Northwest Territories (Giberson et al. 2007 and this study), there does not appear to be an overlap in distribution for the two $H$. pulla forms, so the eastern and western forms may represent different species. The similar Heptagenia solitaria may also be confused in the west with the eastern form of $H$. pulla suggesting that the distribution of the two species should be investigated more thoroughly. Cobb and Flannagan (1980) also noted the presence of Arthroplea bipunctata (McDunnough) (Ephemeroptera: Heptageniidae) and Brachycercus Curtis at one site in the Mackenzie Valley Pipeline study (Martin River; Wiens et al. 1975), although these species were not encountered in the re-examination of the samples (possibly due to the specimen not being retained with other archived samples). The report of these species at only one site suggests that targeted sampling may be needed to clarify their complete distribution in the region. For example, A. bipunctata is usually considered to be more of a lentic rather than a lotic species and has not been reported in either Yukon, Canada or Alaska, United States of America. In contrast, Brachycercus has been reported in Alaska to the west (Randolph and McCafferty 2005).

Species diversity comparisons between re-examined (archived) pipeline study specimens and those from more recent collecting confirmed the value of examining archived specimens, even while emphasising the importance of new collecting. Total numbers of species from the two territories was similar among both sets of samples (Table 3), but there were a number of unique species to each set of samples. Since the pipeline samples date from 1970 to 1973 , they also provide baseline information for comparing species patterns following nearly half a century of climate change and resource development in northwestern Canada. However, since the sampling locations were not the same, differences may relate to different habitats and locations, as well as surveying intensity. For example, although the "newer" collecting in the Northwest Territories included Mackenzie River tributaries that had been sampled in the pipeline study, it also 
included northeastern barrenlands sites in and around the Horton and Thelon Rivers, and mountainous streams in the Mackenzie Mountains west of Norman Wells. Recent sampling was also not as long-term or extensive as the original pipeline study, consisting of "spot" sampling, usually at a single sampling time. The more recent sampling missed many species that were captured in the much more extensive and intensive 1970s monitoring study, even as it produced additional species not encountered in the early study. The 50 species identified from the two territories from these samples included additional territorial records (Northwest Territories: four species plus four tentative species; Yukon: one species plus two tentative species [these are in addition to new records from this study already published in Vinke et al. 2015; Cordero et al. 2016]; see Table 3 for list). One notable record is the presence of Baetis vernus Curtis near Yellowknife in Northwest Territories (and Baetis vernus "cf." in Yukon and the Mackenzie Mountains), since this Palaearctic baetid had not been reported in North America before Cordero et al. (2016).

Recent (post-2000) sampling yielded a substantial number of additional records to the Northwest Territories and Yukon lists, despite being far less intensive than the 1970s pipeline study. The high number of additional species relates to sampling additional regions (including the Mackenzie Mountains and central barrenlands), but also because we targeted capture of large, fresh, and intact specimens that are essential for species-level determination, especially when colour patterns are important in identification. Two groups in particular, Siphlonuridae and Ameletidae, are much easier to identify with newer specimens (particularly in concert with new molecular methods of identification), and continued collecting of these groups should help to determine whether the unidentifiable nymphs in this study are of new or known species. We expect that specimens in two heptageniid genera, Rhithrogena and Cinygmula, from both archived and recent samples may yield several additional species if subsequent taxonomic work results in keys to the nymphs of these species.

Overall, these results demonstrate the value of archived collections to generate updated species lists, and to provide information on distributional information and relative abundances. The challenges to working with archived material (especially if label data is not clear), while daunting, can be overcome to provide baseline and distributional data valuable for conservation efforts, and to provide information on where to target further collecting efforts. New collecting is still needed, however, to obtain identifiable material for current distributional information (using both the updated keys and new techniques such as DNA barcoding). Researchers carrying out large ecological or environmental monitoring studies, particularly in isolated or difficult-to-access locations, should archive samples, with clear label data and associated metadata, to be available for future study.

\section{Acknowledgements}

The authors thank Dr. David Rosenberg (Emeritus Scientist, Freshwater Institute) for the initial push to examine the Mackenzie River pipeline study specimens for comparison with recent studies along the Mackenzie River. Thanks are also due to Laura Rempel for sharing her Ephemeroptera specimens from the Mackenzie River, and to Sarah Tratch (summer student, University of Prince Edward Island) for her tireless work in deciphering the coded labels for the study. Kristen Vinke and Glen Guthrie collected the mayflies near Norman Wells, and Northern Biodiversity Programme samples were collected by Reuben Cordero, Doug Currie, and their field students.

\section{References}

Ball, S.L., Hebert, P.D., Burian, S.K., and Webb, J.M. 2005. Biological identifications of mayflies (Ephemeroptera) using DNA barcodes. Journal of the North American Benthological Society, 24: 508-524.

Brunskill, G.J., Rosenberg, D.M., Snow, N.B., Vascotto, G.L., and Wageman, R. 1973. Ecological studies of aquatic systems in the MackenziePorcupine drainages in relation to proposed pipeline and highway developments. Canada Task Force on Northern Oil Development. Environmental - Social Committee, Northern Pipelines. Volume I: report 73-40 and Volume II appendices: report 73-41. Information Canada, Ottawa, Ontario, Canada.

Buddle, C.M., Currie, D.C., and Giberson, D.J. 2008. Northern insect survey. Newsletter of the Biological Survey of Canada, 26: 63-64.

Catling, P.M., Packer, L., and Goit, M. 2009. COSEWIC insect assessments - processes, achievements and advantages. Newsletter of the Biological Survey of Canada, 28: 66-83. 
Cobb, D.G. and Flannagan, J.F. 1980. The distribution of Ephemeroptera in northern Canada. In Advances in Ephemeroptera biology. Edited by J.F. Flannagan and K.E. Marshall. Plenum, New York, New York, United States of America. Pp. 155-166.

Cobb, D.G., Flannagan, J.F., Flannagan, P.M.L., and Wickstrom, R.D. 1995. Collections of Ephemeroptera from Kluane National Park, Yukon Territory, and Nahanni National Park, Northwest Territories, Canada. In Current directions in research on Ephemeroptera. Edited by L.D. Corkum and J.J.H. Ciborowski. Canadian Scholars Press, Toronto, Ontario, Canada. Pp. 177-184.

Cordero, R.D., Sánchez-Ramírez, S., and Currie, D.C. 2016. DNA barcoding of aquatic insects reveals unforeseen diversity and recurrent population divergence patterns through broad-scale sampling in northern Canada. Polar Biology. doi.org/10.1007/ s00300-016-2062-3.

Currie, D.C., Giberson, D.J., and Adler, P.H. 2002. Insect biodiversity in the Thelon Wildlife Sanctuary. Newsletter of the Biological Survey of Canada, 21: 59-64.

Currie, D.C., Giberson, D.J., and Brown, B.V. 2000. Insects of Keewatin and Mackenzie. Newsletter of the Biological Survey of Canada, 19: 48-51.

Danks, H.V. 2016. The Biological Survey of Canada: a personal history. Biological Survey of Canada Monograph Series 8. Available from http:// biologicalsurvey.ca/monographs/read/19 [accessed 26 February 2017].

Funk, D.H. and Sweeney, B.W. 1994. The larvae of eastern North American Eurylophella Tiensuu (Ephemeroptera: Ephemerellidae). Transactions of the American Entomological Society, 120: 209-286.

Giberson, D.J., Burian, S.K., and Shouldice, M. 2007. Life history of the northern mayfly Baetis bundyae in Rankin Inlet, Nunavut, Canada, with updates to the list of mayflies of Nunavut. The Canadian Entomologist, 139: 628-642.

Gorski, M.R., Fox, A.D., McQueen, J.I., and Jacobus, L.M. 2014. Plauditus cestus (Provonsha \& McCafferty, 1982) (Insecta: Ephemeroptera: Baetidae): new records from Virginia and the Northwest Territories, with notes on color variation. Check List, 11: 1-3.

Guerra García, J.M., Espinosa Torre, F., and García Gómez, J.C. 2008. Trends in taxonomy today: an overview about the main topics in taxonomy. Zoológica Baetica, 19: 15-49.

Harper, F. and Harper, P.P. 1981. Northern Canadian mayflies (Insecta; Ephemeroptera), records and descriptions. Canadian Journal of Zoology, 59: 1784-1789.

Harper, P.P. and Harper, F. 1997. Mayflies (Ephemeroptera) of the Yukon. In Insects of Yukon. Edited by H.V. Danks and J.A. Downes. Biological Survey of Canada Monograph Series 2. Canadian Museum of Nature, Ottawa, Ontario, Canada. Pp. 151-168.
Jacobus, L.M. 2013. A new junior synonym for the Holarctic species Ametropus fragilis Albarda, 1878 (Insecta: Ephemeroptera: Ametropodidae). Proceedings of the Indiana Academy of Science, 122: 18-19.

Jacobus, L.M. and Wiersema, N.A. 2014. The genera Anafroptilum Kluge, 2011 and Neocloeon Traver, 1932, reinstated status, in North America, with remarks about the global composition of Centroptilum Eaton, 1869 (Ephemeroptera: Baetidae). Zootaxa, 3814: 385-391.

Jacobus, L.M., Wiersema, N.A., and Webb, J.M. 2014. Identification of far northern and western North American mayfly larvae (Insecta: Ephemeroptera), north of Mexico; version 2. SAFIT Taxonomic Workshop, Joint Aquatic Science meeting, 3-4 January 2013, Portland Oregon. California State University, Long Beach, California, United States of America.

Mayfly Central 2016. North America species list. Available from: www.entm.purdue.edu/mayfly/naspecies-list.php [accessed 20 February 2017].

McCafferty, W.P., Meyer, M.D., Randolph, R.P., and Webb, J.M. 2008. Evaluation of mayfly species originally described as Baetis Leach (Ephemeroptera: Baetidae) from California. Proceedings of the Entomological Society of Washington, 110: 577-591.

McCafferty, W.P., Meyer, M.D., Webb, J.M., and Jacobus, L.M. 2004. New state and provincial records for North American small minnow mayflies (Ephemeroptera: Baetidae). Entomological News, 115: 93-100.

McCafferty, W.P. and Waltz, R.D. 1990. Revisionary synopsis of the Baetidae (Ephemeroptera) of North and Middle America. Transactions of the American Entomological Society, 116: 769-799.

McCafferty, W.P. and Waltz, R.D. 1995. Labiobaetis (Ephemeroptera, Baetidae) -new status, new North American species, and related new genus. Entomological News, 106: 19-28.

Moore, I.A. 1977. Species descriptions of some Plecoptera, Ephemeroptera, Trichoptera and Diptera from three areas of the Northwest Territories. Volume 77, Environmental Protection Service. Fisheries and the Environment, Northwest Region, Canada.

Morihara, D.K. and McCafferty, W.P. 1979. The Baetis larvae of North America (Ephemeroptera: Baetidae). Transactions of the American Entomological Society, 105: 139-221.

Patterson, D., Mozzherin, D., Shorthouse, D.P., and Thessen, A. 2016. Challenges with using names to link digital biodiversity information. Biodiversity Data Journal, 4: e8080. https://doi.org/10.3897/ BDJ.4.e8080.

Randolph, R.P. 2002. Atlas and biogeographic review of the North American mayflies (Ephemeroptera). Ph.D. dissertation. Department of Entomology, Purdue University, West Lafayette, Indiana, United States of America.

Randolph, R.P. and McCafferty, W.P. 2005. The mayflies (Ephemeroptera) of Alaska, including a new species of Heptageniidae. Proceedings of the Entomological Society of Washington, 107: 190-199. 
Rempel, L.L. and Gill, G.J. 2010. Bioassessment of streams along the Mackenzie Valley using the reference condition approach: biological, habitat, landscape and climate data. Canadian Data Report of Fisheries and Aquatic Sciences 1236. Fisheries and Oceans Canada, Central and Arctic Region, Winnipeg, Manitoba, Canada. Available from www.dfo-mpo. gc.ca/Library/344429.pdf [accessed 26 January 2017].

Robinson, J.L., Fordyce, J.A., and Parker, C.R. 2016. Conservation of aquatic insect species across a protected area network: null model reveals shortfalls of biogeographical knowledge. Journal of Insect Conservation, 20: 565-581.

Stribling, J.B., Moulton, S.R., and Lester, G.T. 2003. Determining the quality of taxonomic data. Journal of the North American Benthological Society, 22: 621-631.

Tancoigne, E. and Dubois, A. 2013. Taxonomy: no decline, but inertia. Cladistics, 29: 567-570.

Vinke, K., Medeiros, A.S., and Giberson, D.J. 2015. Diversity patterns in subarctic stream benthic invertebrate assemblages from the Sahtu Settlement Area, Northwest Territories, Canada. Arctic Science, 1: 9-25.

Waltz, R.D. and Burian, S.K. 2008. Ephemeroptera. In An introduction to the aquatic insects of North America, 4th edition. Edited by R.W. Merritt, K.W. Cummins, and M.B. Berg. Kendall/Hunt Publishing Company, Dubuque, Iowa, United States of America. Pp. 181-236.
Webb, J.M. 2013. A new species of Labiobaetis Novikova \& Kluge, 1987 (Ephemeroptera: Baetidae) from Washington, USA. Zootaxa, 3750: 95-99.

Webb, J.M., Jacobus, L.M., Funk, D.H., Zhou, X., Kondratieff, B., Geraci, C.J., et al. 2012. A DNA barcode library for North American Ephemeroptera: progress and prospects. Public Library of Science One, 7: e38063. https://doi.org/10.1371/journal. pone. 0038063 .

Wiens, A.P., Rosenberg, D.M., and Snow, N.B. 1975. Species list of aquatic plants and animals collected from the Mackenzie and Porcupine River watersheds from 1971-1973. Technical Report 557. Fisheries and Marine Service, Research and Development Directorate, Freshwater Institute, Ottawa, Ontario, Canada. Available from www.dfompo.gc.ca/Library/14967.pdf [accessed 26 January 2017].

Wiersema, N.A., Nelson, C.R., and Kuehnl, K.F. 2004. A new small minnow mayfly (Ephemeroptera: Baetidae) from Utah, U.S.A. Entomological News, 115: 139-145.

Zloty, J. 1996. A revision of the Nearctic Ameletus mayflies based on adult males, with descriptions of seven new species (Ephemeroptera: Ameletidae). The Canadian Entomologist, 128: 293-346. 